\title{
Diaphragmatic Pinch
}

National Cancer Institute

\section{Source}

National Cancer Institute. Diaphragmatic Pinch. NCI Thesaurus. Code C123757.

The location where the diaphrag matic crura constrict the esophagus as it passes through the diaphragm. 